\title{
Alternative Leadership in Flexible Surge Capacity-The Perceived Impact of Tabletop Simulation Exercises on Thai Emergency Physicians Capability to Manage a Major Incident
}

\author{
Phatthranit Phattharapornjaroen ${ }^{1,2}{ }^{\oplus}$, Viktor Glantz ${ }^{1}$, Eric Carlström ${ }^{3,4} \oplus$, \\ Lina Dahlén Holmqvist ${ }^{5}$ and Amir Khorram-Manesh 1,6,*(D) \\ 1 Institute of Clinical Sciences, Department of Surgery, Sahlgrenska Academy, Gothenburg University, \\ 40530 Gothenburg, Sweden; phatthranit.pha@mahidol.edu (P.P.); gusglavi@student.gu.se (V.G.) \\ 2 Department of Emergency Medicine, Center of Excellence, Ramathibodi Hospital, Mahidol University, \\ Bangkok 10400, Thailand \\ 3 Institute of Healthcare Sciences, Sahlgrenska Academy, Gothenburg University, 40100 Gothenburg, Sweden; \\ eric.carlstrom@gu.se \\ 4 USN School of Business, University of South-Eastern Norway, P.O. Box 235, 3603 Kongsberg, Norway \\ 5 Institute of Medicine, Department of Molecular and Clinical Medicine, Sahlgrenska University Hospital, \\ 40530 Gothenburg, Sweden; lina.holmqvist@vgregion.se \\ 6 Department of Research and Development, the Swedish Armed Forces Center for Defense Medicine, \\ Västra Frölunda, 42676 Gothenburg, Sweden \\ * Correspondence: amir.khorram-manesh@surgery.gu.se; Tel.: +46-707-722741
}

Received: 16 July 2020; Accepted: 1 August 2020; Published: 2 August 2020

\begin{abstract}
Flexible surge capacity aims to activate and utilize other resources than normally are surged in a community during the primary and secondary surge capacity. The presence of alternative leadership, skilled and knowledgeable in hospital and prehospital emergency management, is invaluable. Thai emergency physicians work at both levels, emphasizing their important role in emergency management of any source in a disaster-prone country. We aimed to investigate Thai emergency physicians' ability in terms of knowledge and preparedness to manage potential emergencies using tabletop simulation exercises. Using an established method for training collaboration, two training courses were arranged for over 50 Thai emergency physicians, who were divided into three teams of prehospital, hospital, and incident command groups. Three scenarios of a terror attack along with a bomb explosion, riot, and shooting, and high building fire were presented, and the participants' performance was evaluated regarding their preparedness, response and gained knowledge. Two senior observers followed the leadership characteristic in particular. Thai physicians' perceived ability in command and control, communication, collaboration, coordination, and situation assessment improved in all groups systematically. New perspectives and innovative measures were presented by participants, which improved the overall management on the final day. Tabletop simulation exercise increased the perceived ability, knowledge, and attitude of Thai emergency physicians in managing major incidents and disasters. It also enabled them to lead emergency management in a situation when alternative leadership is a necessity as part of the concept of a flexible surge capacity response system.
\end{abstract}

Keywords: disasters; emergencies; flexible surge capacity; leadership; Thailand; 3LC 


\section{Introduction}

Major Incidents and Disasters (MID) affect societies and their inhabitants and result in medical and nonmedical consequences due to the imbalance between the needs and available resources [1]. MID management is a multiprofessional process of organizing and managing the available resources and responsibilities for dealing with all humanitarian aspects of MID, specifically in mitigation, preparedness, response, and recovery phases to lessen the impact of emergencies [2]. Some strategies for MID management include establishing command and control, reliable and efficient ways of communication, information, organization, warning systems, stockpiling of resources, and the development of response plans for the mobilization and management of resources such as personnel, equipment, volunteers, and emergency facilities. The efficiency of the organizational structure in the response is paramount for the outcome of the event $[2,3]$.

The initial approach to proper management of MID is to increase the management system's capacity, i.e., Surge Capacity (SC). Three broad areas of healthcare demand SC in response to a MID [4]. Firstly, Public Health Surge Capacity (the ability of the public health system to increase capacity for patient care, epidemiological investigation, laboratory services, mass fatality management, etc.). Secondly, Healthcare Facility-Based Surge Capacity (augments the response within the healthcare facility structure, e.g., triage-tent on hospital grounds). Finally, Community-based Surge Capacity (the public effort to support and augment the healthcare system). The four essential elements of SC, i.e., Staff, Stuff, Structure, and System (4S), should rapidly and effectively be surged in the affected areas. Staff refers to available/alternative personnel, Stuff refers to available/alternative equipment, Structures refer to Alternate Care Facilities (ACF), and systems are procedures and guidelines that govern the emergency management process [5,6].

The expansion of a MID necessitates a new surge capacity (secondary SC), which underlines the need for extra efforts to obtain additional resources still available within the management system [7-9]. Nevertheless, a further expansion of the incident demands new approaches, policies, and adjustable preparedness within the community to scale up and down resources in a fast, smooth, and productive way, i.e., "flexible surge capacity" (FSC) [10]. The concept of FSC is concordant with the new paradigm of proactivity in disaster management and emphasizes on risk reduction rather than focusing on pure relief operations to reduce vulnerability and increase resilience within communities [11]. Therefore, risk assessment and focus on all four elements of SC is necessary for achieving an FSC. Although the disaster management cycle should incorporate, recognize, and value the participation of affected communities [12], it is necessary that adequate infrastructure is in place to ensure access to emergency services [13]. In this perspective, the readiness of hospitals is essential and among necessary measures, staff pools and new staff categories are needed to replace the regular staffing structure, and to reduce the risk of lacking qualified staff, e.g., leadership.

Although many functions and measures are necessary for proper and successful MID management, the command and control (C2) function remains the most critical function [14,15]. C2 emphasizes the leaderships' characteristics to command, control, communicate, collaborate, and coordinate $[2,6,14,15]$. Such ability offers an excellent opportunity for the leaders to attain a mutual goal, assess the situation from different perspectives, overview all necessary measures, successfully sort out all injuries, treat them adequately and distribute them equally to designated medical facilities. Most hospitals have an emergency management committee, which consists of predesignated positions and staff. Such a group of professionals needs to be trained to act decisively and quickly during crises. Previous studies have, however, shown that these committees suffer from the rarity of MID, and thus, neither have the experience nor are trained enough to act as needed $[15,16]$. There is a lack of experience and knowledge about the management process and collaboration with other partners, particularly in hospital and prehospital arenas. One specialty with the ability and knowledge to connect hospitals and prehospital organizations is Emergency Medicine [17].

Thailand is a disaster-prone country with a westernized healthcare system. Emergency Medicine residency training in Thailand started in 2004, during which the first Tsunami hit its southern part 
and caused mass casualties and structural devastation. One of the outcomes of this deadly event was understanding the needs for organizational and structural changes, and proper knowledge in command and control within the disaster management system. Since then, many educational initiatives such as MIMMS (Major Incident Medical Management and Support), and MRMI (Medical Response to Major Incidents) have been established and yearly conducted to raise the theoretical and practical knowledge of all staff [18-21]. Thai Emergency Physicians (EP), work within and outside the hospitals and are the point of contact and the first line of medical assessment $[19,20]$. This role requires the right level of structural and organizational knowledge of MID management and merits a more responsible and critical role in response to major emergencies. One way to obtain such knowledge is through tabletop simulations training [21].

The Three-Level Collaboration (3LC) training model is used to train small groups of commanders up to hundreds of participants in the management of MID. The development of the 3LC model was based on hypothesizing that the collaborative elements in a mutual task help reduce the organizational barriers [21]. The method has been compared with traditional exercises in several studies [22-24]. In one of these, the 3LC method improved collaboration as well as learning. At the 3LC exercise, $94.3 \%$ of the personnel perceived the exercise to be focused on collaboration. The traditional exercises reported a corresponding figure of $75.6 \%$. The majority of the participants answered that they learned something new during the exercises (78.5\%, traditional $64.9 \%$ ). They also felt that the exercises had an impact on real-life activities during daily work (80.4\%, traditional 61.7\%) [24]. Organizational capabilities and limitations are enlisted to promote interplay with no hierarchical authority, and to promote the ability to switch between different collaboration strategies as demanded by the specific situation. Collaboration training offers a chance to not only exhibit stability (the quality that one develops through drill and practice), but also to practice transitions, overlaps, fearlessness, improvisation, creative thinking, and the ability to handle unexpected situations. Such education is beyond the repeated learning that comes from the drill in control and command structures and other mechanical structures.

\section{Aim}

This study aimed to evaluate the progress of knowledge and learning outcomes of a group of Thai EPs before and after 3LC simulation exercise in seven documented and essential areas (CSCATTT): Command and control, Safety, Communication, Triage, Treatment, and Transport, which according to MIMMS concept are essential elements to lead and manage a MID [18].

\section{Method}

\subsection{Population}

All EPs voluntarily registered to an Incident Command System course at a major hospital in Bangkok, Thailand. Participants who were present during the entire course session were included. Consequently, those who could not present or left the course for a few hours were excluded. Otherwise, there were no exclusion criteria, and all staff working with MID, including nurses could take part in the course.

\subsection{Course Design}

Two two-day courses with the same structure consisting of a short introduction and a scenario simulation exercise on the first day, followed by two more scenarios on day two. A round of discussion and comments for the whole course took place at the end of the course. Participants were divided into three groups of (1) a prehospital team (Operational), (2) emergency departments command group (Tactical), and (3) hospital command group (Strategical).

There was a rotation between different groups, which enabled all participants to try different positions to reach consensus in the whole group of participants to achieve similarities rather than differences. No instruction was given about participants' posts in each group, and they had to figure 
out what they need and how to use all competences, based on the given lectures and earlier knowledge in MIMMS. Three different scenarios (1) fire in a tall building, (2) a terrorist attack and a bomb explosion, and (3) riots, including shooting, were presented to the groups during rotation (Appendix A). After each scenario, the participants in each group had to present their results. Each group received comments from supervisors and other participants. At the end of the presentations, and according to the 3LC model, the participants in each group had to gather again and discuss what they could do better if they had a new opportunity. Each group presented the results of this round, and other participants and supervisors could comment on their findings. Finally, each group gathered to discuss the necessary knowledge and functions and guidelines for each position to be taken home as the quality, which is needed for operational, tactical, and strategic levels. Besides, two supervisors followed each group and evaluated their performance based on MIMMS-CSCATT concept (see below) [18].

\subsection{Pre- and Post-Course Test}

The pre- and post-course tests were conducted to evaluate the gained knowledge in each participant [25]. The tests were designed using the learning objectives established for the course, i.e., crucial factors for successful management of MID incorporated in CSCATTT (Command and control, Security, Communication, Assessment, Triage, Treatment, Transport), a well-known model used in MIMMS courses internationally [18]. Both tests consist of the same items. The test aimed for each participant's self-assessment in knowledge about the subjects of CSCATTT, displayed as a Likert scale, in which No knowledge was represented by $0 \%$, while $100 \%$ was full knowledge. Participants were asked to mark the grade of their understanding in each topic on a five-level Likert Scale. A nonparametric test, Wilcoxon signed rank test, was chosen to compare the means.

\subsection{Observational Evaluation}

All groups were observed based on a Participant Observation model with a focus on the leadership in all groups [26-28]. Among various signs of good leadership, the following points were mainly observed to evaluate the leadership: Communication and Decision-making ability, Accountability, Delegation, and Empowerment of others. Two observers collected and noted their observations, which were discussed after each scenario to reach a consensus. The observer registered if a commander was identified and appointed during the incident and if the appointed commander possessed the control. The observer did also register if the commander ensured successful medical management, i.e., built a team of multiple staff overcoming a potentially chaotic situation and if the team reached a subtactical, tactical, or operational level [18]. The data were recorded as Event Sampling, which allows focusing on one specific subject. All other types of behavior were ignored [26-28].

\subsection{Observational Evaluation}

As part of 3LC simulation training, group discussions took place after each scenario. Besides confirming and discussing the results and improvement measures, these discussions also aimed to discuss the observational findings in each occasion. The process aimed to pair data collected by observation to the discussion and interviews to unearth individual motivations or behaviors that were not immediately obvious in a group setting [24-28].

\section{Results}

There were 56 participants registered, however, only 52 could complete the course. The four physicians excluded (two females and two males) had to go back to work due to emergencies, and were not presented at all levels of simulation training. A sum of 52 EPs took part in the entire course on two occasions. Nurses were also invited to these courses, but none were registered due to hesitance of the spoken language of the course (English). There were 36 females and 16 males. The age of the participants ranged between 26-35 years, with a range of experience between 1-10 years as EP. The majority of participants were from the hospitals in Bangkok. However, 16 physicians were from 
hospitals in other parts of Thailand. The pre- and post-training tests were conducted before and after the session.

a. Command and control (C2): There was an increasing knowledge of $\mathrm{C} 2$ after the course. Around $20 \%$ of the EPs $(n=10)$ had little or very little knowledge about C2, and $46 \%(n=24)$ had medium knowledge. At the end of the course, there was a shift to the positive end, where the majority of our participants had a good to a very good understanding of C2 function ( $n=49,94 \%)$ (Figure 2). The change was statistically significant $(p=0.00, z=-5.436)$.

b. Safety: There was an increasing knowledge of safety issues after the course. Around $15 \%$ of the EPs $(n=8)$ had no or very little knowledge about safety issues, and $44 \%(n=23)$ had medium knowledge. At the end of the course, there was a shift to the positive end, where the majority of our participants had a good to a very good understanding of safety issues $(n=50,96 \%)$ (Figure 3 ). The change was statistically significant $(p=0.00, \mathrm{z}=-6.056)$.

c. Communication: There was an increasing knowledge of communication ways and difficulties after the course. Around $21 \%(n=11)$ of the EPs had none or very little knowledge about communications issues, and $44 \%(n=23)$ had medium knowledge. At the end of the course, there was a shift to the positive end, where the majority of our participants had a good to a very good understanding of safety issues $(n=48,92 \%)$ (Figure 4$)$. The change was statistically significant $(p=0.00, \mathrm{z}=-5.872)$.

d. Assessment: There was an increasing knowledge about the importance of the assessment during a major incident after the course. Around $17 \%(n=9)$ of the EPs had no or very little knowledge about assessment and its association with the incident management, and $48 \%(n=25)$ had medium knowledge. At the end of the course, there was a shift to the positive end, where the majority of our participants had a good to a very good understanding of assessment issues $(n=48,92 \%)$ (Figure 5). The change was statistically significant $(p=0.00, z=-5.464)$.

e. Triage: There was an increasing knowledge about the importance of triage and associated dilemmas during a major incident after the course. Around $17 \%(n=9)$ of the EPs had no or very little knowledge about triage and its role during incident management, and 30\% $(n=16)$ had medium knowledge. Although 29 EPs reported a good or very good knowledge, there was still a shift to the positive end at the end of the course, where the majority of our participants had a good to a very good understanding of triage issues $(n=46,88 \%)$ (Figure 6). The change was statistically significant $(p=0.00, \mathrm{z}=-4.585)$.

f. Treatment: There was an increasing knowledge about the treatment alternatives and options at the right time and right place during a major incident after the course. Around $17 \%(n=9)$ of the EPs had none or very little knowledge about the treatment given during incident management, and $28 \%(n=15)$ had medium knowledge. Although 28 EPs reported good or very good knowledge, there was still a shift to the positive end at the end of the course, where the majority of the participants had good to a very good understanding of triage issues $(n=37,71 \%)$ (Figure 7). The change was statistically significant $(p=0.00, \mathrm{z}=-5.287)$.

g. Transport: There was an increasing knowledge about the importance of triage and associated dilemmas during a major incident after the course. Around 21\% $(n=11)$ of the EPs had none or very little knowledge about triage and its role during incident management, and $32 \%(n=17)$ had medium knowledge. At the end of the course, there was a shift to the positive end, where the majority of our participants had a good to a very good understanding of assessment issues $(n=38,73 \%)$ (Figure 8). The change was statistically significant $(p=0.00, z=-5.634)$.

h. Observations: Two critical observations were reported (Figure 1). Both observers collected their documented results in which they had specifically commented on the ability of decision-making, communication, accountability, empowerment of other group member and delegation of tasks. Consensus was made and the groups were exposed to the observers' understanding to unearth individual motivations or behaviors that were not immediately obvious in a group setting [24,25]. 
a. During the exercises, each team selected its leader with three leadership styles, without any previous knowledge and spontaneously;

i. $\quad$ Consensus leadership (G3): The leader tried to reach consensus with other members before any decision. Slower decision-making process.

ii. $\quad$ Passive leadership (G2): The leader laid back and observed other members and only interrupted if needed. Decisions were made, but sometimes not of the leader.

iii. Active Leadership (G1): The leader had the last word in every decision and continuously directed the group. Less delegation and empowerment.

b. Each group developed its communication method consisting of a mobile telephone, paper-based messages, and signs.

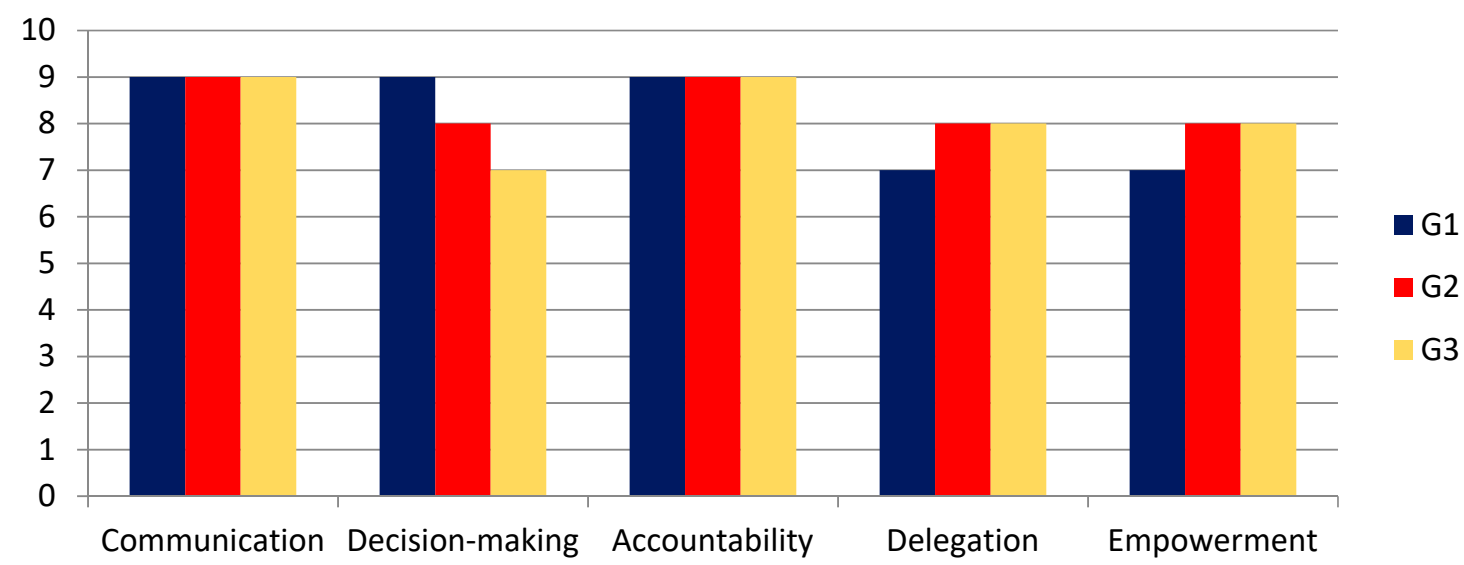

Figure 1. Shows the results of the leadership characteristics during the exercise.

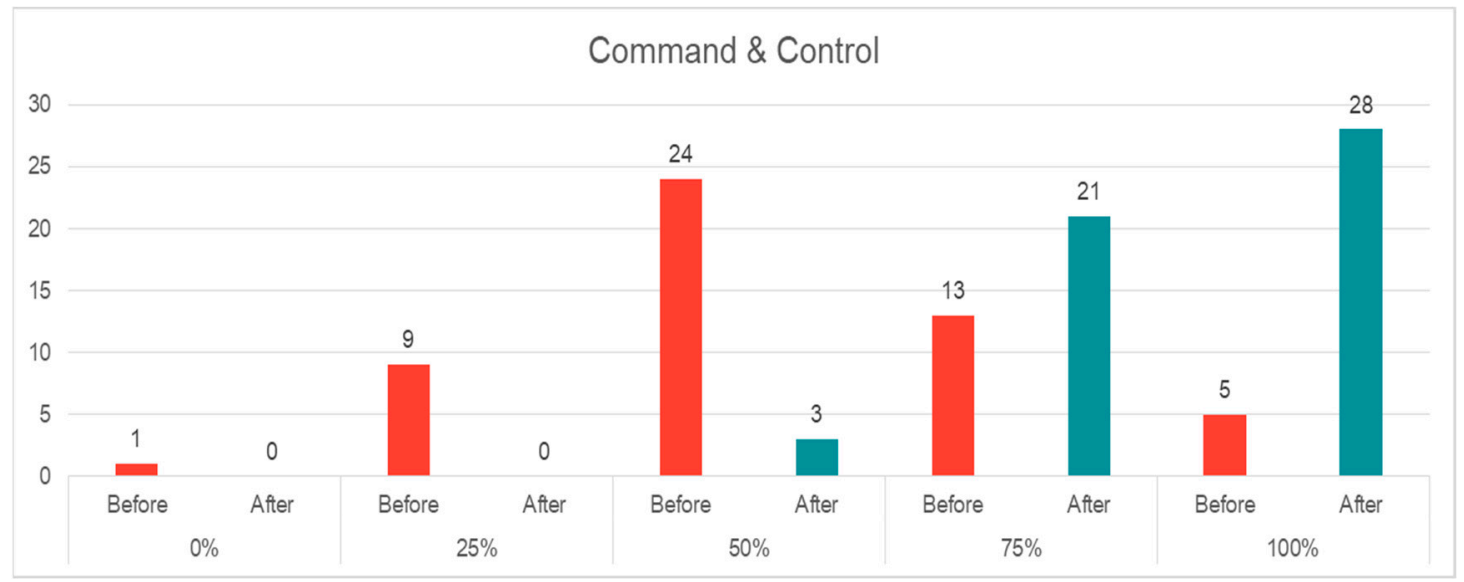

Figure 2. Shows the results of the pre-, and post-test in Command and Control. 


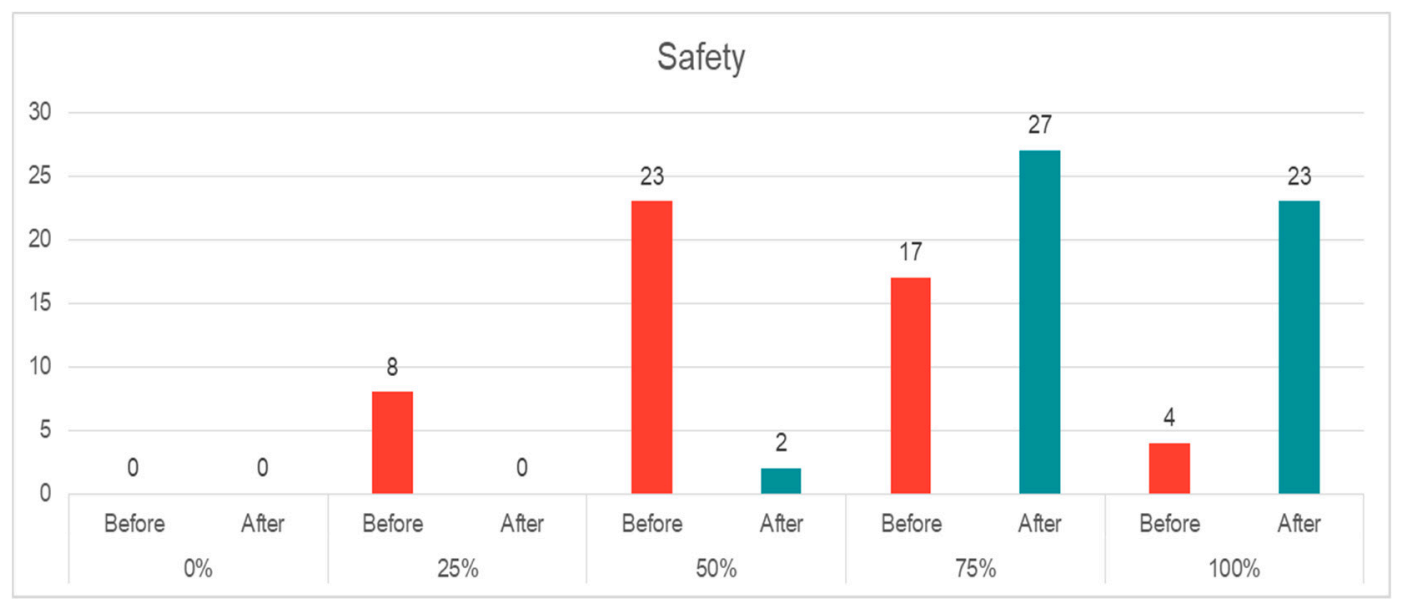

Figure 3. Shows the results of the pre-, and post-test in Safety issues.

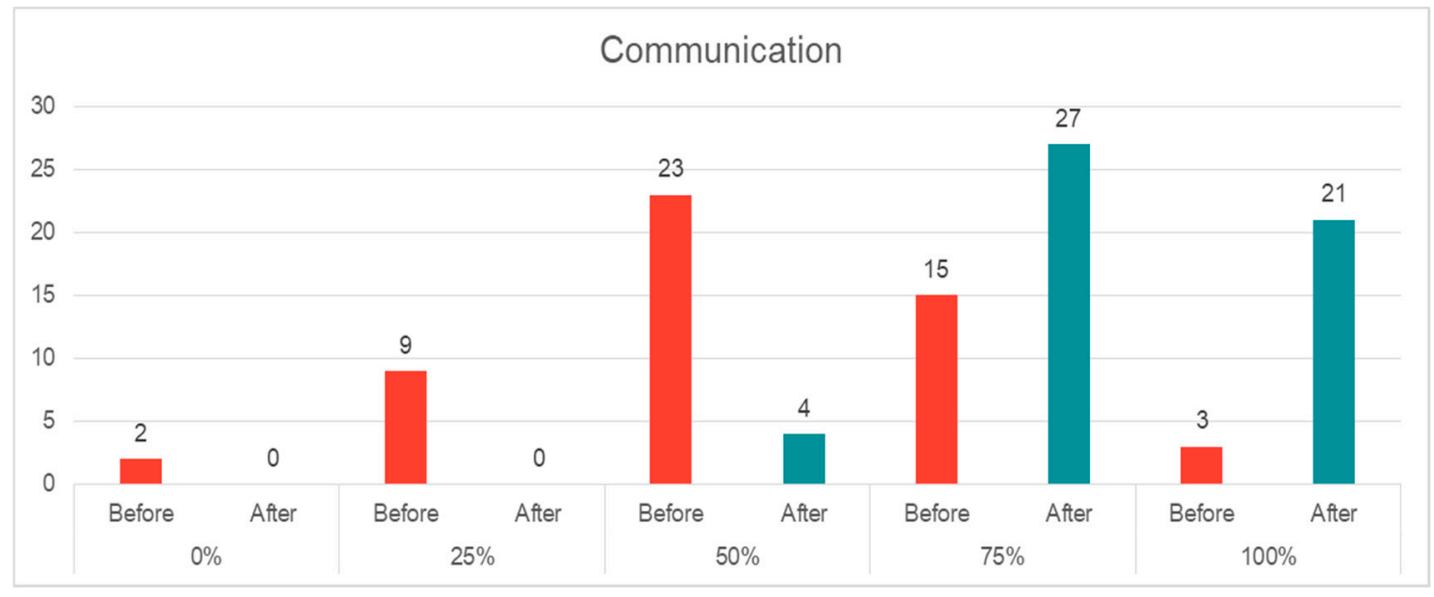

Figure 4. Shows the results of the pre-, and post-test in Communication.

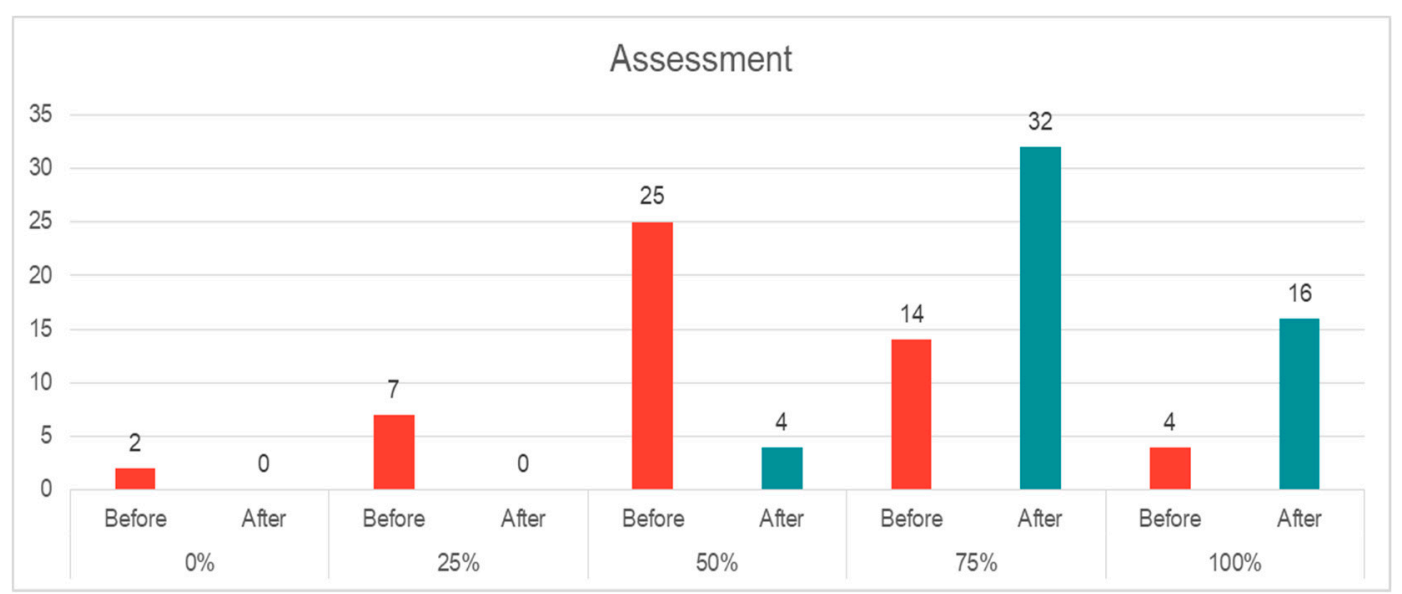

Figure 5. Shows the results of the pre-, and post-test in situation Assessment. 


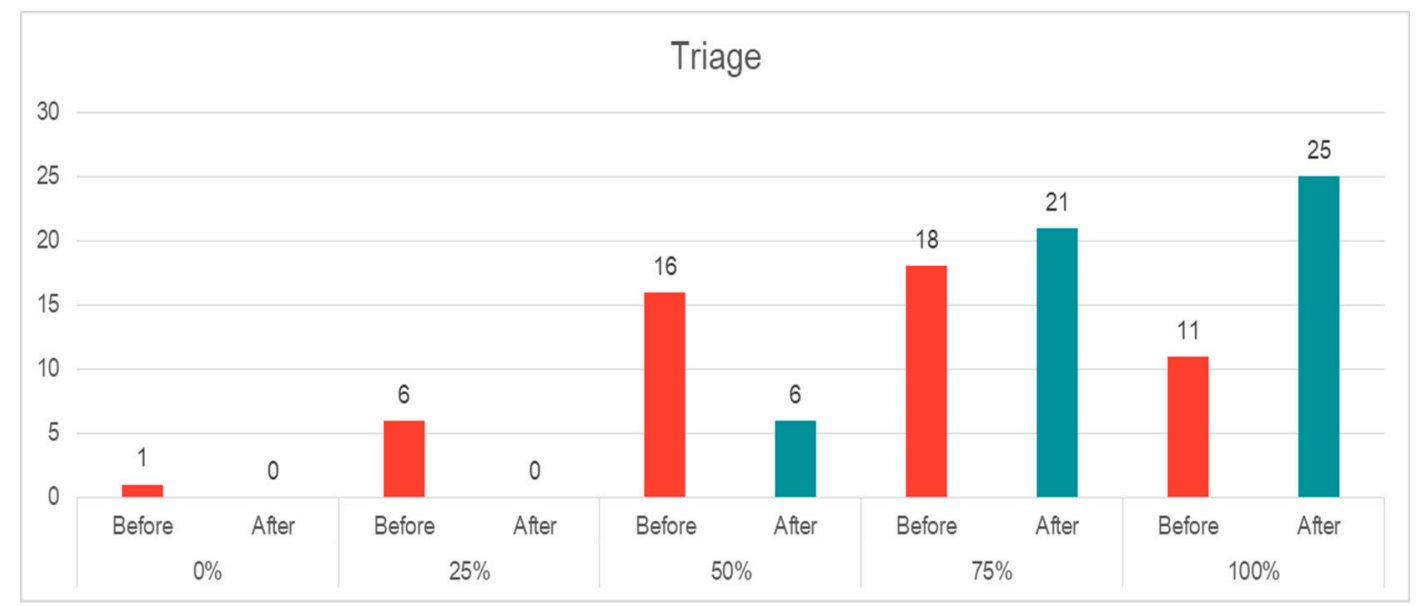

Figure 6. Shows the results of the pre-, and post-test in Triage.

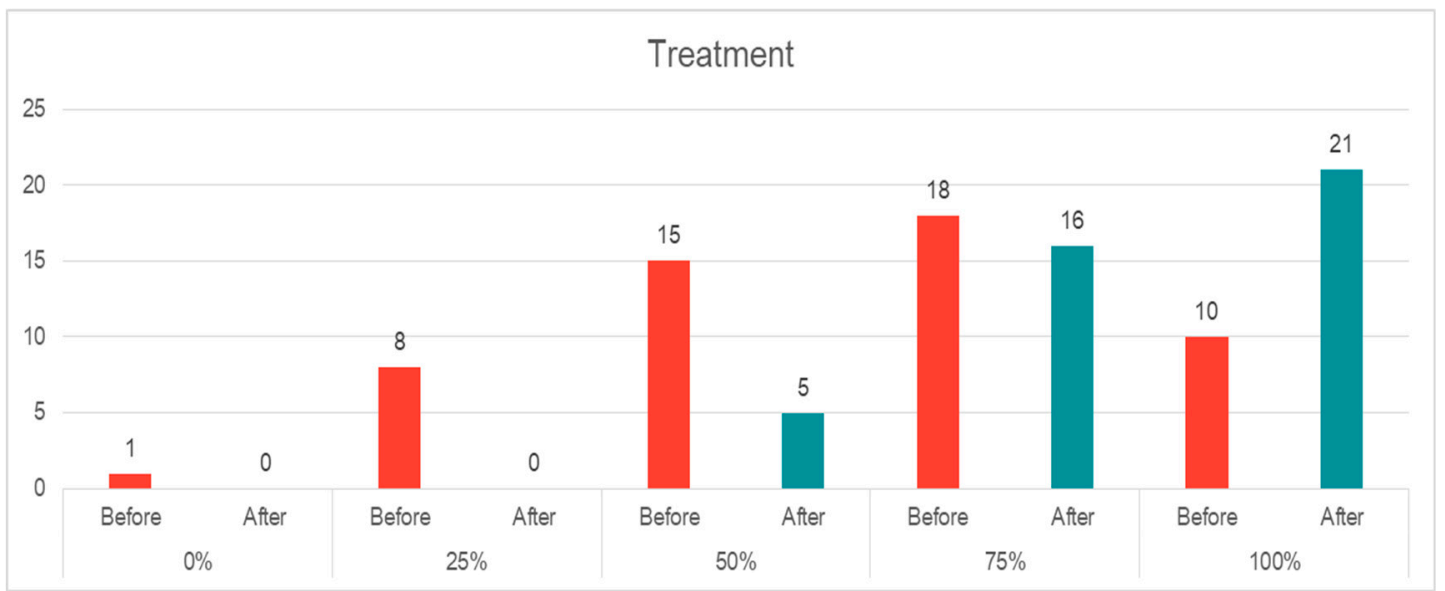

Figure 7. Shows the results of the pre-, and post-test Treatment issues.

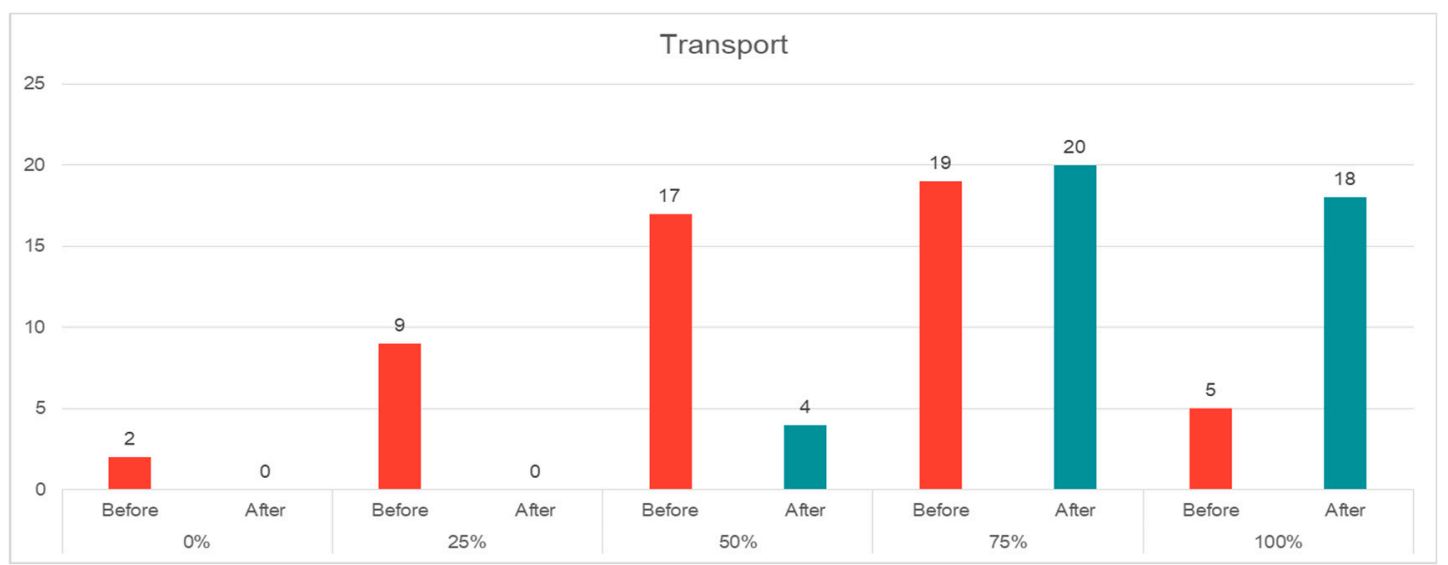

Figure 8. Shows the results of the pre-, and post-test Transport issues.

\section{Discussion}

The essential raise of knowledge among Thai EPs was registered in Command and control, Safety, Communication, and situation Assessment. At the same time, the understanding of the subject areas, Triage, Treatment, and Transport did not develop to the same extent. All changes were statistically significant. Since these physicians are working at both hospital and prehospital levels, it is not surprising 
that the grade of their knowledge in strict medical areas, e.g., Triage, Treatment, and Transport, is higher than other subjects within CSCATTT, which specifically deal with leadership characteristics.

Earlier studies have discussed the medical and nonmedical aspects of MID management, showing critical shortcomings in the latter [15]. Disaster and major incidents are rare, but they need to be mitigated and managed if they occur. In successful management, collaboration is one crucial factor that demands skilled leadership and reliable communication. In this group of EPs with no earlier experience of MID, the collaborative elements in a mutual task helped to reduce the organizational barriers, enlisted their abilities and limitations, and promoted interplay with no hierarchical authority, as well as the ability to switch between different collaboration strategies as demanded by the specific situation and leadership styles. The groups grew up to be more stable, practiced transitions, overlaps, fearlessness, and improvised to create new ways of handling the unexpected situation by discovering different communication alternatives. During training, their ability to try out the situation developed their confidence, increased their trust to each other, and reduced the internal fear of doing wrong. They could test a model, evaluate its effect in the next stage, create a new approach, test it, and develop their approach continuously $[21,23,24,29]$. All these elements are necessary for successful teamwork at the time of emergency. The ultimate requirement is to act in a flexible surge capacity (FSC) when hospitals and communities must activate their resources, respectively [10].

Choosing EPs as one of the major players in MID management is a natural selection $[17,19,20]$. In countries such as Thailand, they work at both hospital and prehospital levels and thus have a logical connection to both entities $[19,20]$. In a changing world, when resources may not be enough to match the needs, there must be more material and personal options than what is offered by standard disaster plans. FSC aims to provide new innovative solutions to these shortcomings [10]. Other reports have high lightened the need for FSC and discussed the need for ACF [30]. This paper tried to examine the possibility of using a simulation model to increase the knowledge and understanding of a new set of professionals to lead MID management. Training EPs for the next MID will strengthen the whole chain of disaster management and partly accommodate the WHO's policy of proactivity and increase the strength and ability of communities to handle the first waves of MID impacts since EPs are one group, who will face upcoming incidents $[17,19]$. Other measures within the community, such as civilians' empowerment as immediate responders, and the necessity of creating new guidelines and systems have been reported elsewhere [31-33]. Altogether, they facilitate the concept of FSC (Flexible Surge Capacity). Using the elements of CSCATTT as a model enables a direct assessment of critical features that need to be in place during MID management [18]. These elements are part of educational initiatives given to all healthcare staff, and are not only perceived abilities and knowledge but also what they practice in every-day missions, and emphasize the importance of medical and nonmedical aspects of MID management. In a future with limited resources and expanding needs, civilian help can be decisive in creating Resilience in each society [31-33]. The same method and elements can be used to educate other groups of people to act at different levels of management or maybe increase their awareness about any given event to mitigate the adverse outcomes of any incidents [15,30-34].

Moynihan [29] emphasizes the value of trying it out, i.e., to test a model to cope with an event, evaluate the effect of the model in the next stage, create a new approach, test it, and develop the method continuously. Through the 3LC-exercise, the EPs practiced their ability to make a concerted and coherent assessment of which collaboration form is applicable at a given time. The collaboration is not a static activity but subject to construction, deconstruction, and reconstruction, depending on what is appropriate for a specific time, place, or event. The 3LC exercise illustrates the primary goal of practicing the different forms of collaboration and switching between them by including asymmetries in the scenarios [21-24]. For instance, the police can arrive first at the scene and begin caring for victims with serious injuries, or understaffed emergency rescue services have to get help from the police and paramedics at a fire incident. The exercise also reveals the need for a joint assessment and the importance of knowing both prehospital and hospital fields. The latter offers a valuable opportunity to 
increase the collaborative segment of MID management. At the same time, decisions can be made based on knowledge about the different organizations' limitations and capabilities [15,16].

\section{Limitations}

This study did not compare the 3LC and traditional types of exercises. In earlier studies, 3LC had a significant impact on perceived collaboration, learning as well as performance. Based on these findings, before and after design control was chosen (pre- and post-test) [22-25]. Measuring the perceived effects of exercises, using a self-assessment tool, may be considered as a limitation. The possibility of over- or underreporting cannot be ignored. However, there are some advantages with the tool too, such as it encourages the participants to reflect over their own learning progress and performance, to be more responsible for their own learning, to develop their judgment skills while they have no pressure of peer evaluation, make participants more autonomous learners and finally make them aware of their weaknesses and strength. To verify the causality and generalizability of the results, a study that is not just based on a written test would give additional insightful and important information.

As an alternative, a comparative design, including a control group, should be considered in future studies. However, pre- and post-test scores provide information on whether or not participants have learned from the training. Besides, a well-designed pre- and post-test can help trainers understand which concepts or competencies were well taught during the training and which ones need additional time, or need to be covered using alternative methods $[25,33]$. Pre- and post-tests may not be the best tools to use for every type of training but still offer a good overview of the tested group's advancement. Wishing to understand what knowledge can be credited to the training itself, using a pre- and post-test methodology is essential. As shown statistically in this study, the knowledge of EPs in all areas of CSCATT increased significantly. Another limitation was that the data collection occurred immediately after the exercises and not supplemented with data collected weeks or months after the exercises [27,28].

An alternative method of evaluating learning, such as the observation of skills demonstrated in a role-play, might be more appropriate in some situations. In this study, we combined the Participant observation method as a complement to the pre- and post-course tests [26-29]. It is a variant of the natural observations model. Still, the researcher joins in and becomes part of the group they are studying to get a more in-depth insight into their activities. In this overt observation model, the researchers revealed their identity and purpose to the group and asked permission to observe. There are some limitations to this model. It can be challenging to get time/privacy for recording, which means observers have to wait until they are alone and rely on their memory, which is a problem as they may forget details and are unlikely to remember direct quotations. Furthermore, if the researcher becomes too involved, they may lose objectivity and become bias, and thus reduce the validity of the data.

The participants in this study were all physicians, while other staff such as nurses, paramedics, administrators, etc. are also important parts of a hospitals' MID management. This study was the first step in a series of studies that are planned to evaluate the concept of FSC, and physicians were simply chosen due to their actual position within the Thai healthcare and their knowledge in English. It is clear that other knowledgeable staff could also possess the leadership position [34].

Finally, although statistically significant results were obtained in this study, the number of included EPs can be challenged. However, for everyone working within healthcare, it is clear that a larger number of physicians cannot be gathered at the same time for any kind of exercise or training. This is a challenging fact that affects all types of education and training within all healthcare systems $[21,35]$. Similar argument is true concerning the provision of a control group. It is hard to get enough healthcare workers in a training session, and still harder to have one third of them as a control group. Simulation exercises emerged as a solution to this issue since reality-based exercises demand not only a large number of participants but also cost a lot and often evaluate just one part of the disaster management chain $[21,35]$. 


\section{Conclusions}

In conclusion, the findings in this report indicate that the ability in all elements of CSCATTT, particularly expertise in command and control, communication, collaboration and coordination, and situation assessment, improved in all groups of EPs systematically and after each scenario. The 3LC method also enabled all participants to create new perspectives and innovative measures, which improved the overall management of scenarios on the final day. The 3LC increases the ability, knowledge, and attitude of Thai emergency physicians in managing MID. It also enables them to lead emergency management in an unexpected situation, as alternative leaders in a flexible surge capacity-response system [10,30].

Author Contributions: Conceptualization, A.K.-M.; data curation, P.P.; formal analysis, P.P., V.G., E.C. and A.K.-M.; investigation, P.P. and A.K.-M.; methodology, E.C. and A.K.-M.; project administration, P.P.; resources, P.P., A.K.-M.; supervision, A.K.-M.; validation, L.D.H.; writing—original draft, P.P.; writing—review \& editing, V.G., E.C., L.D.H. and A.K.-M. All authors have read and agreed to the published version of the manuscript.

Funding: This research received no external funding.

Acknowledgments: The authors would like to thank Yohan Robinson for his support to this project.

Conflicts of Interest: The authors declare no conflict of interest.

\section{Appendix A}

Appendix A.1. Scenarios, 3LC Exercises, Bangkok, Thailand

\section{Scenario 1: A bomb}

On 15 September 2017, the dispatch center contacted and informed you about a Bomb found $5 \mathrm{~km}$ from your hospital. There was another hospital $200 \mathrm{~m}$ next to the scene. More than 100 people were injured. Mange situation based on CSCATTT.

Around $30 \mathrm{~min}$ after the first contact, a second bomb is found at the middle of the scene. How would you manage the scene now?

\section{Scenario 2: A Fire in a Tall Building}

On 15 December 2017, the dispatch center contacted and informed you that there was a fire on the fifth floor of an 11 floors apartment $5 \mathrm{~km}$ away from your hospital. More than 50 people were injured. How do you manage the situation based on CSCATTT?

Around $30 \mathrm{~min}$ after the fire incident and when there is a crowd and gathering of people, a truck drives into the treatment zones inside the area, and hit many people.

\section{Scenario 3: Riot and Active Shooter}

On 15 May 2015, you were on a night shift at the hospital and saw Riots on the news; the place of the incident was $5 \mathrm{~km}$ from your hospital. Some people starts shooting and ambulances started and kept bringing injured people to your hospital. How would you manage your emergency department based on CSCATTT?

One of the injured victim had a bomb strapped on his chest. How would you manage the situation? Would you evacuate your emergency department? 


\section{References}

1. WHO. Hospital Emergency Response Checklist. An All-Hazards Tool for Hospital Administrators and Emergency Managers. 2011. Available online: http://www.euro.who.int/_data/assets/pdf_file/0008/268766/ Hospital-emergency-response-checklist-Eng.pdf (accessed on 25 June 2020).

2. Vitalii, S.; Khorram-Manesh, A.; Nyberg, L. Disaster cycle and management. In Handbook of Disaster and Emergency Management; Khorram-Manesh, A., Ed.; Kompendiet: Göteborg, Sweden, 2017; Chapter 2; pp. 23-29.

3. United Nations International Strategy for Disaster Reduction (UNISDR). Terminology on Disaster Risk Reduction; United Nations: Geneva, Switzerland, 2009. Available online: https://www.unisdr.org/files/7817_ UNISDRTerminologyEnglish.pdf (accessed on 25 June 2020).

4. Hick, J.; Christian, M.; Sprung, C. Surge Capacity and Infrastructure considerations for mass critical care. Intensive Care Med. 2010, 36, 11-20. [CrossRef]

5. Barbich, D.; Koenig, K. Understanding Surge Capacity: Essential Elements. Acad. Emerg. Med. 2006, 6, 1098-1102. [CrossRef] [PubMed]

6. Kaji, A.; Koenig, K.L.; Bey, T. Surge capacity for healthcare systems: A conceptual framework. Acad. Emerg. Med. 2006, 13, 1157-1159. [CrossRef] [PubMed]

7. Bonnet, C.; Peery, B.; Cantrill, S.; Pons, P.; Haukoos, J.; McVaney, K.; Colwell, C. Surge capacity: A proposed conceptual framework. Am. J. Emerg. Med. 2007, 25, 297-306. [CrossRef] [PubMed]

8. Runkle, J.D.; Brock-Martin, A.; Karmus, W.; Svendsen, E.R. Secondary Surge Capacity: A framework for understanding long-term access to primary care for medically vulnerable populations in disaster recovery. Am. J. Public Health 2012, 102, 24-32. [CrossRef] [PubMed]

9. Adams, L.M. Exploring the concept of surge capacity. Issues Nurs. 2009, 14, 2. [CrossRef]

10. Khorram-Manesh, A. Flexible Surge Capacity_Public Health, Public Education, and Disaster Management. Health Promot. Perspect 2020, 10, 175-179. [CrossRef]

11. The Lancet Editorial. Disaster prevention should be equal. Lancet Glob. Health 2017, 5, 1047. [CrossRef]

12. Krolik, M. Exploring a rights-based approach to disaster management. Aust. J. Emerg. Manag. 2013, 28, 44-48. Available online: https://knowledge.aidr.org.au/resources/ajem-oct-2013-exploring-a-rights-basedapproach-to-disaster-management/ (accessed on 14 July 2020).

13. Rice, K.; Felizzi, M.V.; Hagelgans, D. Human Rights-Based Approach to Disaster Management: Valparaiso, Chile. J. Hum. Rights Soc. Work 2017, 2, 117-127. [CrossRef]

14. Prytz, E.G.; Rybing, J.; Carlström, E.; Khorram-Manesh, A.; Jonson, C.O. Exploring prehospital C2-work during a mass gathering event. Int. J. Emerg. Serv. 2015, 4, 227-241. [CrossRef]

15. Khorram-Manesh, A.; Lönroth, H.; Rotter, P.; Wilhelmsson, M.; Aremyr, J.; Berner, A.; Nero Andersson, A.; Carlström, E. Non-medical aspects of civilian-military collaboration in management of major incidents. Eur. J. Trauma Emerg. Surg. 2017, 43, 595-603. [CrossRef]

16. Khorram-Manesh, A.; Hedelin, A.; Örtenwall, P. Regional coordination in medical emergencies and major incidents; plan, execute and teach. Scand. J. Trauma Resusc. Emerg. Med. 2009, 17, 32. [CrossRef]

17. Kahn, C.A.; Koenig, K.L.; Schultz, C.H. Emergency physician disaster deployment: Issues to consider and model policy. Prehosp. Disaster Med. 2017, 32, 462-464. [CrossRef]

18. Sammut, J.; Cato, D.; Homer, T. Major Incident Medical Management and Support (MIMMS): A practical multiple casualty, disaster-site training course for all Australian healthcare personnel. Emerg. Med. 2001, 13, 174-180. [CrossRef]

19. Sittichanbuncha, Y.; Prachanukool, T.; Sarathep, P.; Sawanyawisuth, K. An emergency medical service in Thailand-Providers perspectives. J. Med. Assoc. Thai 2014, 97, 1016-1021.

20. Khorram-Manesh, A.; Angthong, C.; Pangma, A.; Sulannakarn, S.; Burivong, R.; Jarayabhand, R.; Örtenwall, P. Hospital Evacuation; Learning from the Past? Flooding of Bangkok 2011. BJMMR 2014, 4, 395-415. [CrossRef]

21. Khorram-Manesh, A.; Berlin, J.; Carlström, E. Two validated ways of the ability of decision-making in emergencies, Results from a literature review. Bull. Emerg. Trauma 2016, 4, 186-196.

22. Sorensen, J.; Magnussen, L.I.; Torgersen, G.E.; Christiansen, A.M.; Carlstrom, E. Percieved Usefulness of Maritime Cross-Border Collaboration Exercises. Arts Soc. Sci. J. 2018, 9, 1-5. [CrossRef] 
23. Magnussen, L.I.; Carlström, E.; Sörensen, J.L.; Torgersen, G.E.; Hagenes, E.F.; Kristiansen, E. Learning and Usefulness stemming from collaboration in a maritime crisis management exercise in Northern Norway. Disaster Prev. Manag. 2018, 27, 129-140. [CrossRef]

24. Berlin, J.; Carlström, E. The three level collaboration exercise-Impact of learning and usefulness. J. Conting. Crisis Manag. (JCCM) 2015, 23, 257-265. [CrossRef]

25. Aykal, G.; Kespali, M.; Aydin, Ö.; Esen, H.; Yegin, A.; Gungör, F.; Yilmaz, N. Pre-Test and Post-Test Applications to Shape the Education of Phlebotomists in a Quality Management Program: An Experience in a Training Hospital. J. Med. Biochem. 2016, 35, 347-353. [PubMed]

26. Takala, E.P.; Pehkonen, I.; Forsman, M.; Hansson, G.Å.; Mathiassen, S.E.; Neumann, W.P.; Sjögaard, G.; Veiersted, K.B.; Westgaard, R.H.; Winkel, J. Systematic evaluation of observational methods assessing biomechanical exposures at work. Scand. J. Work Environ. Health 2010, 36, 3-24. [CrossRef] [PubMed]

27. Luna-Reyes, L.F.; Lines Andersen, D. Collecting and analyzing qualitative data for system dynamics: Methods and models. Syst. Dyn. Rev. 2004. [CrossRef]

28. Lofland, J.; Lofland, L.H. Analyzing Social Setting: A Guide to Qualitative Observation and Analysis, 2nd ed.; Wadsworth: Belmont, CA, USA, 1984.

29. Moynihan, D.P. Learning under uncertainty: Networks in crisis management. Public Admin. Rev. 2008, 68, 350-365. [CrossRef]

30. Glantz, V.; Phattharapornjaroen, P.; Carlström, E.; Khorram-Manesh, A. Regional Flexible Surge Capacity-A flexible response system. Sustainability 2020, 12, 5984. [CrossRef]

31. World Health Organization (WHO). Sustainable Development Goals (SDGs). Available online: https: //www.who.int/sdg/en/ (accessed on 25 June 2020).

32. Khorram-Manesh, A.; Plegas, P.; Peyravi, M.; Carlström, E. Immediate Response to Major Incidents: Defining an immediate responder! Eur. J. Trauma Emerg. Surg. 2019. [CrossRef]

33. Khorram-Manesh, A.; Berlin, J.; Ljung Roseke, L.; Aremyr, J.; Sörensson, J.; Carlström, E. Emergency Management and Preparedness Training for Youth (EMPTY): The Results of the First Swedish pilot Study. Disaster Med. Public Health Prep. 2018, 12, 685-688. [CrossRef]

34. Khorram-Manesh, A. Learning to Respect Diversity, Gender Equality, and Collaboration: A childhood Agenda or the Content of a Leadership Development Program; Magnussen, L.I., Ed.; Disaster, Diversity, and Emergency Preparation; IOS Press: Amsterdam, The Netherlands, 2019; pp. 11-18. [CrossRef]

35. Jahangirian, M.; Naseer, A.; Stergioulas, L.; Young, T.; Eldabi, T.; Brailsford, S.; Patel, B.; Harper, P. Simulation in health-care: Lessons from other sectors. Oper. Res. Int. J. 2012, 12, 45-55. [CrossRef] 